\title{
RESEARCH OF IMPLICIT ATTITUDES TOWARDS GAMBLING FOR GAMBLERS AND NON-GAMBLERS
}

\author{
Irina Plotka \\ Baltic International Academy, Latvia \\ Nina Blumenau \\ Baltic International Academy, Latvia \\ Zhanna Vinogradova \\ Baltic International Academy, Latvia
}

\begin{abstract}
The importance of studying attitudes towards gambling has been recently recognized in the field of gambling. Research aim is to examine whether non-gamblers and gamblers exhibit both positive and negative implicit attitudes towards gambling-related stimuli. Research questions: (I) What is the valence of implicit associations with gambling among gamblers and non-gamblers? (II) Are the differences in attitudes towards gambling revealed by explicit and implicit methods among gamblers and non-gamblers? (III) Is there a consistency between results obtained by implicit measures and explicit measures of attitude towards gambling? Methods: Participants - 98, age 18-58, Mdn=34 years. Groups: Social Gamblers - 24, Problem Gamblers - 25, Non-Gamblers - 49. Implicit measures: Modified version of Single-Category Implicit Association Test (SC-IAT). Explicit measures: "Gambling Attitudes and Beliefs (GABS)" and "Gamblers anonymous twenty questions (GA-20)". Results and Conclusions. (I) Both positive and negative implicit gambling associations were found in each of the groups. (II) Explicit attitudes towards gambling are most pronounced in social gamblers and most not pronounced in non-gamblers. Differences in implicit associations with gambling among the groups were not found. (III) In case of positive implicit associations, a negative correlation between the results of explicit and implicit measurements was found. It is possible that the use of negative implicit associations will contribute to the classification of gamblers with low, moderate and high risk.
\end{abstract}

Keywords: attitudes towards gambling, implicit attitudes, implicit association test, explicit measure, problem gamblers, social gamblers.

\section{Introduction}

Numerous researches are aimed at better understanding the nature of gambling, as well as to develop effective intervention strategies to prevent the progress of problematic gambling.

Major study of problem gamblers, including their profile, attitudes and gambling behavior. There is no widely accepted causal explanation or single theoretical model that adequately accounts for the etiology of problem gambling. Learning theory, cognitive models, and neurophysiologic models all 
have some evidence base. Very little evidence supports personality or psychoanalytic explanations. Integrated models comprising biopsychosocial and pathways approaches are supported by emerging evidence, leading to a framework identifying at least three primary subgroups of gamblers: behaviorally conditioned, emotionally vulnerable, and biologically-based impulsive (Rickwood, Blaszczynski, Delfabbro, Dowling \& Heading, 2010, p. 4).

The use of narrative literature reviews allowed the researchers to distinguish various self-report and indirect procedures to assess craving for gambling, including single-item rating scales, multi-item questionnaires, freeresponse verbalization tasks, physiological measures, and reaction time (RT) tasks (Ashrafiuon \& Rosenberg, 2012, p. 538). There are various questionnaires aimed to determine the level of gambling involvement. The most commonly used are self-assessment procedures, such as Canadian Problem Gambling Index (CPGI) (Ferris \& Wynne, 2001), the South Oaks Gambling Screen (SOGS) (Lesieur \& Blume, 1987), Victorian Gambling Screen (VGS) (Ben-Tovim, Esterman, Tolchard \& Battersby, 2001) or DSM-IV, which are sufficiently valid and reliable. For the purpose of the research the authors used the self-report questionnaire Gamblers Anonymous Twenty Questionnaire (GA-20). In terms of validity the GA-20 was highly correlated with the SOGS ( $\mathrm{r}=0.94)$, both instruments have a number of items that are virtually identical. There are numerous self-assessment measures that can be used to assess attitudes towards gambling. Often used questionnaires are Gamblers Beliefs and Attitude Survey (GABS) (Breen \& Zuckerman, 1999) and Attitude Towards Gambling Scale (ATGS) (Orford, Griffiths, Wardle, Sproston \& Erens, 2009). In recent years, in connection with intensive development of implicit methodology the new models to explain addictive behavior have appeared. Assessment of implicit processes has been accompanied by new dual-process models of addictive behaviors (Wiers \& Stacy, 2006). According to dual process models, addictive behaviors are determined by the dynamic interaction of two different qualitative processes: the fast, automatic "impulsive system" and the slow, deliberative "reflective system" (Strack \& Deutsch, 2004). The reflective system is responsible for carrying out processes of rule-based reasoning and of symbolic representation. The impulsive system refers to "a network in which information is processed automatically through a fast and parallel spread of activation along the associative links between contents" (Strack \& Deutsch, 2004, p. 208). Attitudes towards gambling have been mostly measured with explicit methods, although it has been stated that explicit attitude measures could have significant limitations due to the social desirability. Especially, when measuring such a socially sensitive topic as attitudes towards gambling, participants could be motivated not to report the true attitudes. Among the most increasingly popular there are 
implicit measurements, such as the Implicit Association Test and its variants, as well as the emotional unconscious priming. They may also reveal information that is not available to introspective access and that conflicts with reachable cognition (Nosek, Greenwald \& Banaji, 2007), and this further makes them resistant to self-deception (Ackermann \& Teichert, 2015). Contemporary psychological research widely uses both explicit and implicit methods for measuring attitudes. The terms ,explicit” and ,implicit” relate to cognitive constructs and processes measured by various procedures.

Explicit methods are direct, controlled, and conscious. They are based on explicit knowledge about oneself and often do not reveal the true but socially desirable answers of participants. Implicit methods are indirect, automatic, and unconscious. Implicit methods are measurements of automatic (unconscious) evaluative reactions that come to mind spontaneously, with one presentation of the object towards which the attitude has been formed (Petty, Fazio \& Brinol, 2009, pp. 3-8). They are based on the measurement of the reaction time of participants when they perform a variety of tasks and their attention is focused on performing these tasks, rather than on the object of attitude. In implicit social cognition the definition of attitude is understood as a mental link between the object of attitude and its final evaluations (attributes) which are stored in memory (Fazio, 2007). A popular definition of implicit attitudes is "introspectively unidentified traces of past experience that mediate favorable or unfavorable feeling, thought, or action towards social objects" (Greenwald \& Banaji, 1995). Even though this definition of implicit attitudes is often interpreted as implicit measures of attitudes that provide "unconscious" access to attitudes, recent studies show that individuals often realize that they possess the attitude that is being assessed by response time-based measures (De Houwer, 2006).

Achievements in implicit social cognition enable us to consider psychological constructs not only on the controlled, but also on the automatic level. Attitudes towards gambling are estimated with implicit measurement techniques as well. However, the investigation of automatic memory processes may be of huge relevance for the study of the psychology of gambling (Yi \& Kanetkar, 2010).

There is a number of recent studies on automatic activation of gambling related non-evaluative associations (Ackermann \& Teichert, 2015, Yi \& Kanetkar, 2010). One of the reasons explaining this growing interest is the fact that implicit measures have been proven to overcome social desirability biases (Ackermann \& Mathieu, 2015). In a gambling-specific Stroop task study Boyer and Dickerson (2003) reported that gamblers who suffer impaired control took significantly longer to name the color of the words related to gambling, whereas this finding was not observed among the gamblers of high control. These 
findings suggest that gamblers have a high activation potential of gamblingrelated associations in their memory due to frequent gambling (Yi \& Kanetkar, 2010, p. 141). Zack \& Poulos $(2004,2007)$ studied the priming effect of an amphetamine on automatic activation of the gambling concept. The priming of the amphetamine significantly increased problem gamblers' response to gambling words in the Lexical Salience Task (LST), but it inhibited their responses to neutral words. As expected, the amphetamine's selective activation of gambling words was not observed among non-problem gamblers. These different studies demonstrate that implicit measures, i.e. the Stroop task and priming tasks are a valid and valuable tool to tap gambling associations, and that the strength of gambling associations stored in memory vary among problem gamblers and non-problem gamblers (Ackermann \& Teichert, 2015, Yi \& Kanetkar, 2010, Ashrafioun \& Rosenberg, 2012).

One of the studies on attitudes towards gambling using implicit measures has been conducted by $\mathrm{Yi}$ and Kanetkar (2010). It investigated weather individuals with a greater risk of gambling problems will have more positive implicit attitudes than those with lower gambling risk. It was found that SC-IAT procedure is a bipolar measure of attitudes in which participants are asked to map their attitudes towards gambling in the positive-negative bipolar dimension. In the Brevers' study (Brevers, et al., 2013) there were two forms (positive and negative) of modified ST-IATs used. The results of the study showed that in comparison to non-gamblers, problem gamblers exhibit positive, but not negative, implicit attitudes towards gambling. It can be assumed that a difference in the research results can be explained by different samples of research participants, as well as the specifics of various IAT measurements.

This paper presents a study using SC-IAT method to research attitudes towards gambling on the sample of problem gamblers, social gamblers and nongamblers. In order to select the categories of gamblers the self-report questionnaire Gamblers Anonymous Twenty Questionnaire (GA-20) was used. As the self-assessment procedure which measures attitudes towards gambling the authors used Gambling Attitude and Beliefs Survey (GABS) (Breen \& Zuckerman, 1999). The present research is a continuation of the research (Plotka, 2013) which involved only social gamblers and non-gamblers.

Research aim is to examine whether non-gamblers and gamblers exhibit both positive and negative implicit attitudes towards gambling-related stimuli.

\section{The Research questions are:}

(I) What is the valence of implicit associations with gambling among gamblers and non-gamblers?

(II) Are the differences in attitudes to gambling revealed by explicit and implicit methods among gamblers and non-gamblers? 
(III) Is there a consistency between the results obtained by implicit measures and explicit measures of attitude towards gambling?

\section{Method}

Participants - 98, age 18-58, Mdn = 34 years. Groups: Social Gamblers 24, Problem Gamblers - 25, Non-Gamblers - 49. The native language of all of the participants was Russian.

"Social gamblers" were selected from gamblers who visit casinos at least once a week. All of them belong to category of "non-problem social gamblers", who are employed or learning. Social gamblers consider gambling to be a valid form of recreational activity and maintain full control over the time, money and energy they expend on gambling. They consider the cost of gambling to be payment for entertainment (Davis, 2003). "Problem Gamblers" and "NonGamblers" groups were performed due to "GA-20". A problem gambler is a subject who has frequent repeated episodes of gambling that dominate the subject's life and leads to a decrease in social, occupational, material and family values, neglecting responsibilities in this sphere.

\section{Explicit methods:}

Breen and Zuckerman's Self-reported procedure Gambling Attitudes and Beliefs (GABS) (Breen \& Zuckerman, 1999). The GABS is a 35-item, 4point scale. GABS items were constructed to capture a wide range of positive evaluation of gambling, cognitive biases and irrational beliefs, attitudes, and characterizing behaviors. The GABS had undergone an initial adaptation process and was modified for Russian speaking audience. The translation of the method to Russian was performed using a reverse translation method: the GABS translation in Russian was translated further back to English by an independent translator. After this the original text of GABS was compared with the final translation in English and the differences between the translation and the original were discussed and adjustments were made for the final text of GABS Russian version. The GABS showed acceptable internal consistency (coefficient alpha $=0.70)($ George \& Mallery, 2003). The GABS is a 35-item, 4-point scale. GABS items were constructed to capture a wide range of positive evaluation of gambling, cognitive biases and irrational beliefs, attitudes, and characterizing behaviors. According to the authors, all the items of GABS could be loaded on one big factor, which can be represented as a general affinity to gambling. Therefore, the GABS can evaluate individuals who, at the moment, are not pathological gamblers, but who may have cognitive risk factors that indicate the possibility of becoming a problem-gambler (Breen \& Zuckerman, 1999). The levels are: 0-35 - no dependence, 36-70 moderate desire to gambling, 71-105 dependence, 106-140 - heavy dependence on gambling. 
Gamblers anonymous twenty questions - GA-20 in a modified version of A.A. Karpov, V.V. Kozlov (2012), which allowed the researchers to classify the participants by categories „Problem Gamblers” and „Non-Gamblers”. GA uses a set of 20 items, commonly referred to as the GA-20, for the purpose of indicating whether someone is a compulsive (problem) gambler. The questions include content such as remorse over gambling, gambling to forget problems, borrowing money to gamble, and difficulty sleeping. Endorsing seven or more questions indicates that the person is likely a compulsive gambler. The internal consistency of the GA-20 was Cronbach`s alpha $=0.94$, indicating high internal consistency. In terms of validity the GA-20 was highly correlated with the SOGS ( $\mathrm{r}=0.94)$, and the authors noted that the both instruments have a number of items that are virtually identical.

Implicit method: A modified version of Single-Category Implicit Association Test (SC-IAT), developed on the basis of SC-IAT (Karpinski \& Steinman, 2006, Yi \& Kanetkar, 2010). SC-IAT procedure was adapted in order to assess the non-relative implicit attitude toward gambling.

The categories of all SC-IAT were: in verbal form - "game for money", in visual form - six pictures with a gambling theme, covering different types of gambling.

The attributes - the words with a strong affective meaning (positive or negative) were used.

"Unpleasant" attributes: Terrible, Bad, Humiliating, Calamitous, Hideous, Catastrophic, Abusive, Discouraging, Disappointing, Nasty, and Gloomy.

"Pleasant" attributes: Awesome, Excellent, Wonderful, Marvelous, Excellent, Pleasant, Grandiose, Attractive, Amazing, Fascinating, and Impressive.

Apparatus: Certified licensed software E-Prime $2 ®$.

Research procedure. All the participants took part in the research voluntarily. The research was conducted individually. Participants completed the tasks in the same order: SC-IAT measure and explicit measures. At the conclusion of the session the participants were thanked and completely debriefed.

SC-IAT measure. The authors applied the experimental procedure SCIAT, using six trial blocks. Performance of the implicit method took an average of 10 to 20 minutes. Participants' reaction time (RT) was registered. Each stage was preceded by a set of instructions concerning the dimensions of the categorization task and the appropriate key responses. Instructions were written in black letters on a white background and located in the center of the monitor screen. Each sentence began with a new line. Each target word appeared centered on the screen. All target and category words were presented in lowercase letters. Before the start of the experiment, on a computer monitor a participant was 
given general instructions and specific instructions before each of the blocks (tasks). The task of the participants was the differentiation of presented stimuli. The gambling SC-IAT combined verbal and visual stimulus (6 pictures with gambling theme, covering different types of gambling). Stimulus word displayed on the screen without auditory accompaniment and remained on the screen until the pressing a key of the participant (Table 1). The RT for each trail was recorded as the time interval between the onset of stimulus presentation and pressing the correct key. Words were selected randomly without replacement.

Table 1 Single-Category IAT for Gambling

\begin{tabular}{|c|c|c|c|c|}
\hline Block & Trials & Function & Left-key response „Q" & Right-key response „P” \\
\hline 1 & 30 & Practice & $\begin{array}{l}\text { Pleasant words }+ \\
+ \text { gambling }(\mathrm{w})\end{array}$ & Unpleasant words \\
\hline 2 & 34 & Test & $\begin{array}{l}\text { Pleasant words + } \\
+ \text { gambling(v) }\end{array}$ & Unpleasant words \\
\hline 3 & 34 & Test & $\begin{array}{l}\text { Pleasant words }+ \\
+ \text { gambling }(\mathrm{v})\end{array}$ & Unpleasant words \\
\hline 4 & 30 & Practice & Pleasant words & $\begin{array}{l}\text { Unpleasant words + } \\
+ \text { gambling }(\mathrm{w})\end{array}$ \\
\hline 5 & 34 & Test & Pleasant words & $\begin{array}{l}\text { Unpleasant words + } \\
+ \text { gambling }(\mathrm{v})\end{array}$ \\
\hline 6 & 34 & Test & Pleasant words & $\begin{array}{l}\text { Unpleasant words + } \\
+ \text { gambling }(\mathrm{v})\end{array}$ \\
\hline
\end{tabular}

Note. $v$-visual category, $w$-verbal category.

The target word remained on the screen until the participants responded.

To ensure the internal validity of the experiment the main parameters were unchanged (the time of stimulus presentation, the intervals between stimuli, number of stimuli - the words, the font, chromatic background settings).

\section{Results}

Explicit measured variable "GABS" was measured with Breen and Zuckerman's Gambling Attitudes and Beliefs Scale.

Implicit measured variable. As a result of SC-IAT the D-scores (effect size) for implicitly measured variable "Gambling implicit associations" were calculated (Rudman, 2011):

$$
D=\frac{1}{2}\left(\frac{M_{5}-M_{2}}{S D_{52}}+\frac{M_{6}-M_{3}}{S D_{63}}\right),
$$

where $M_{i}$ is the mean of RT in block " $i$ ", $S D_{i k}-$ is combined standard deviation for blocks " $i$ " and " $k$ ". 
All trials with RT $<400 \mathrm{~ms}$ and RT $>10000 \mathrm{~ms}$ were deleted.

The D-statistic is an effect size, based on each person's variance in response latencies. If $|\mathrm{D}| \leq 0.15$ - no effect, if $0.15<|\mathrm{D}| \leq 0.35-\mathrm{p}$, if $0.35<|\mathrm{D}|<0.60$ - medium effect size, if $|\mathrm{D}| \geq 0.60$ - large effect size.

Statistical methods. With research of descriptive statistics, extreme values of variables and compliance of data distribution with normal distribution it was found that variables can be researched by methods of parametric statistics, using t-tests for means, Pearson's correlation coefficients and ANOVA.

The first research question. The following statistical methods were used.

Frequencies analysis.

Both positive and negative implicit gambling associations were found in each of the groups (Fig. 1-6).

Group

Social Gamblers

\section{Problem}

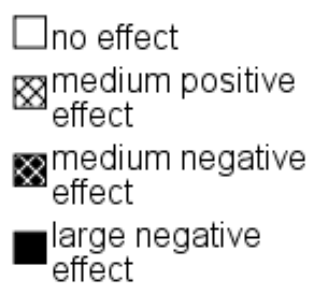

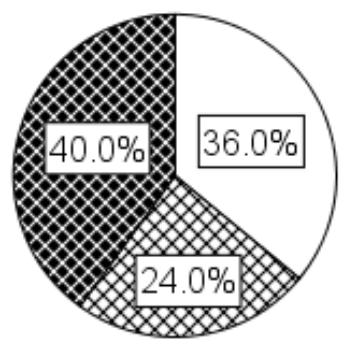
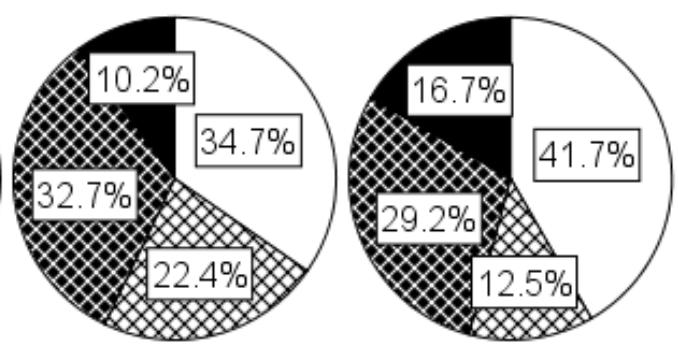

Figure 1 Implicit associations toward gambling effects in percent for social gamblers, problem gamblers and non-gamblers

The differences in the ratios between the percent of participants with positive and negative associations were tested using Fisher's Angle Transformation $\varphi^{*}$-test. Social Gamblers: Medium negative effect $40.0 \%$, medium positive effect $24.0 \%, \varphi^{*}=1.22$, ns; Non-Gamblers: Medium \& high negative effect $42.9 \%$, medium positive effect $22.4 \%, \varphi^{*}=2.19, p<.05$; Problem Gamblers: Medium \& high negative effect $45.9 \%$, medium positive effect $12.5 \%, \varphi^{*}=2.65, p<.01$. Both in the group of non-gamblers, and in the group of problem gamblers the percentage of participants with negative associations with gambling is significantly higher than the percentage of participants with positive associations. Among social gamblers the differences in percentage of players with positive and negative associations were not found. 


\section{Two - Samples t-test}

The statistically significant difference between the mean of positive $\mathrm{D}$ $\left(\mathrm{M}\left(\mathrm{D}^{+}\right)\right)$in "Social Gamblers" and $\mathrm{M}\left(\mathrm{D}^{+}\right)$in "Non-Gamblers" was not found, $\mathrm{t}(30)=-0.85$, ns. The statistically significant difference between the mean of positive $\mathrm{M}\left(\mathrm{D}^{+}\right)$in "Social Gamblers" and $\mathrm{M}\left(\mathrm{D}^{+}\right)$in "Problem Gamblers" was not found, $\mathrm{t}(23)=1.30$, ns. The $\mathrm{M}\left(\mathrm{D}^{+}\right)$in "Non-Gamblers" exceeds the $\mathrm{M}\left(\mathrm{D}^{+}\right)$in "Problem Gamblers": $\mathrm{t}(30)=2.79, \mathrm{p}<.01$. Among problem gamblers the positive implicit effect is less than that of non-gamblers (Fig. 2).

The statistically significant difference between mean of negative $\mathrm{D}$

$\left(\mathrm{M}\left(\mathrm{D}^{-}\right)\right)$in "Social Gamblers" and $\mathrm{M}\left(\mathrm{D}^{-}\right)$in "Non-Gamblers" was not found, $\mathrm{t}(39)=0.53$, ns. The $\mathrm{M}\left(\mathrm{D}^{-}\right)$in "Non-Gamblers" exceeds the $\mathrm{M}\left(\mathrm{D}^{-}\right)$in "Problem Gamblers": $\mathrm{t}(38)=2.16, \mathrm{p}<.05$. The $\mathrm{M}\left(\mathrm{D}^{-}\right)$in "Social Gamblers" exceeds the $\mathrm{M}\left(\mathrm{D}^{-}\right)$in "Problem Gamblers": $\mathrm{t}(14)=2.30, \mathrm{p}<.05$. Problem gamblers have the most pronounced negative effect (Fig. 2).

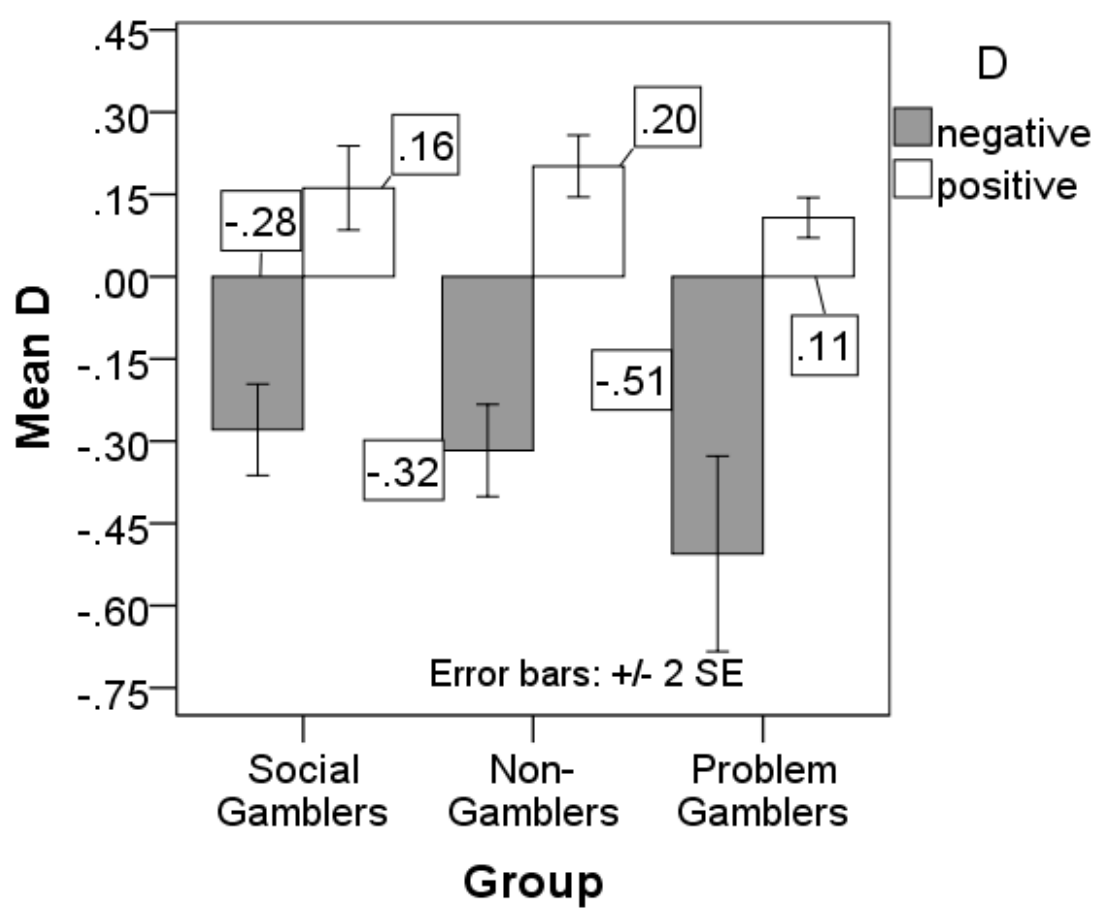

Figure 2 Means of positive and negative D-scores for each of groups

\section{One-Sample t-test}

Difference between mean of D and zero in "Social Gamblers" is not found: $\mathrm{t}(23)=-1.10$, ns. Positive and negative effects are counterbalanced. In "NonGamblers" $\mathrm{t}(48)=-2.30, \mathrm{p}<.05$ and in "Problem Gamblers" $\mathrm{t}(23)=-2.29, \mathrm{p}<.05$ outweighs the negative effect (Fig. 3). 


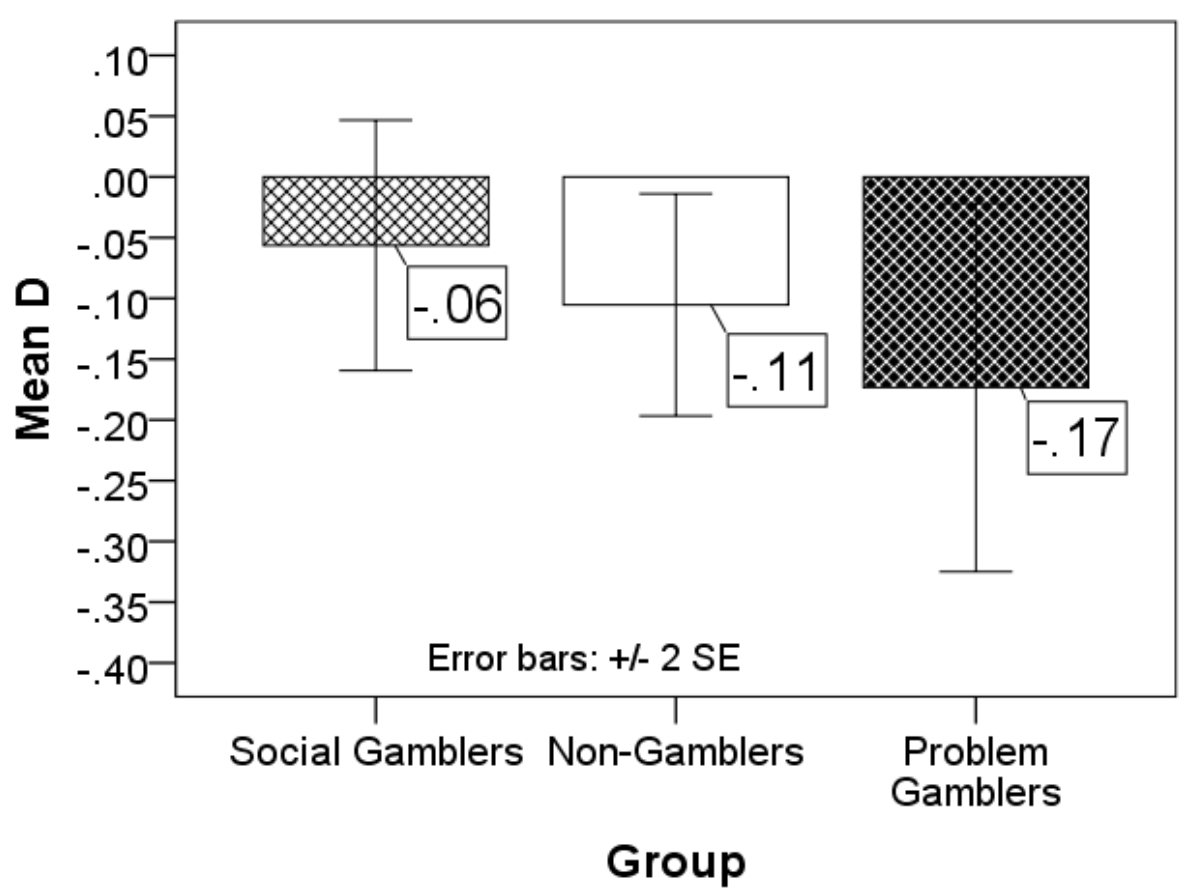

Figure 3 Means of $\mathbf{D}$ for each group

On the horizontal axis in Fig. 4-6 there are the codes of participants, on the vertical axis - implicitly measured associations with gambling (D) and the values of explicit measured variable GABS, divided by 200 (for visual clarity). The levels for GABS/200 are: 0-0.175 - no dependence, 0.18-35 moderate desire to gambling, 0.355-0.525 - dependence, 0.53-0.70 - heavy dependence on gambling.

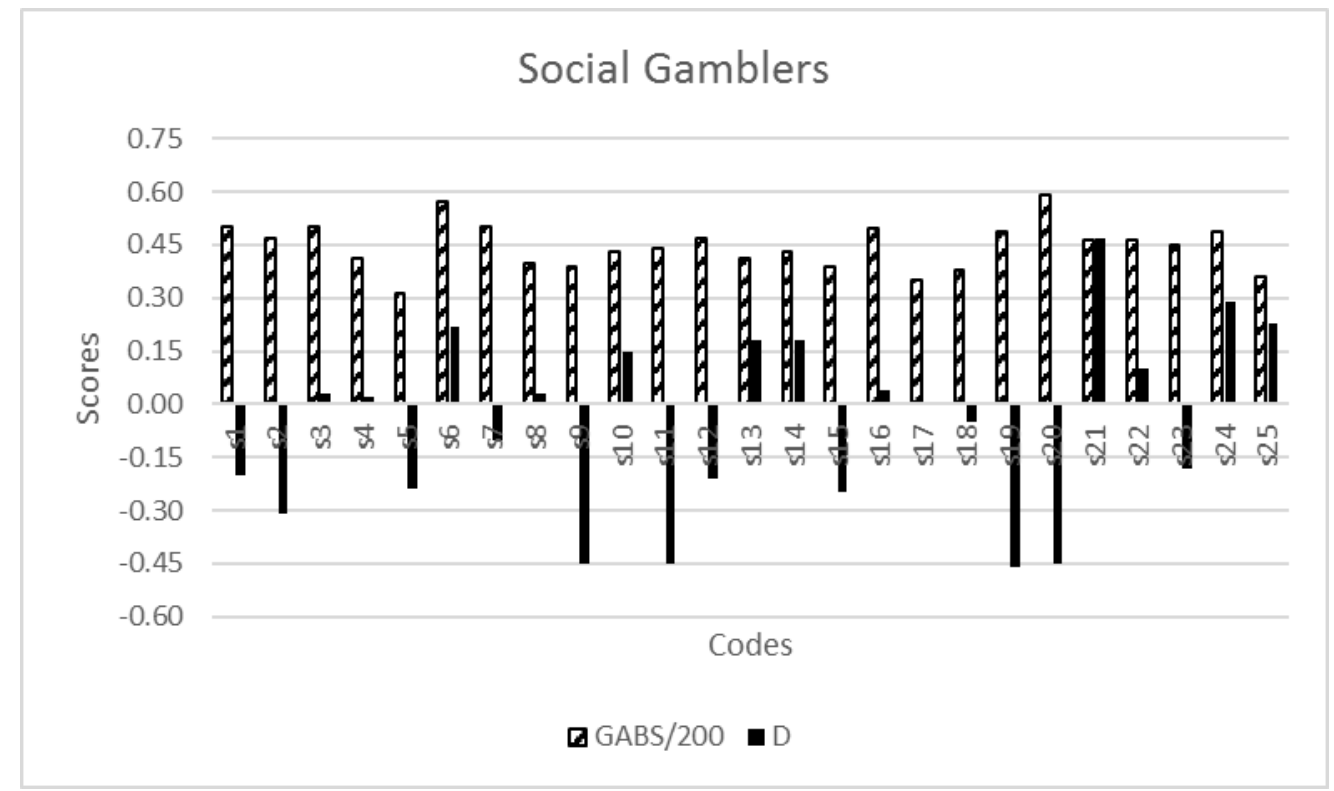

Figure 4 Results of attitudes to gambling measures with explicit method GABS/200 and D. Group "Social gamblers" 
Proceedings of the International Scientific Conference. Volume I, May $27^{\text {th }}-28^{\text {th }}$, 2016. 498-514

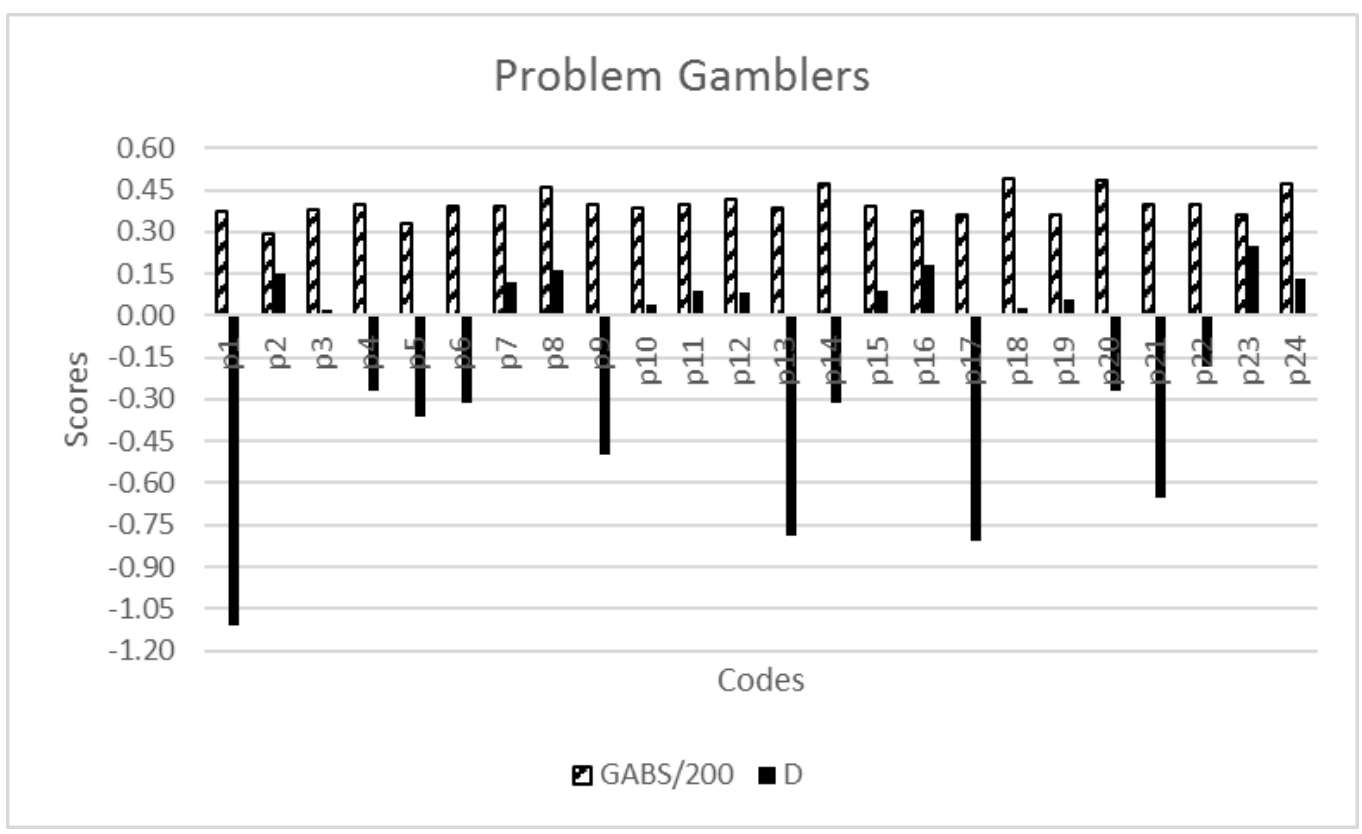

Figure 5 Results of attitudes to gambling measures with explicit method GABS/200 and D. Group "Problem gamblers"

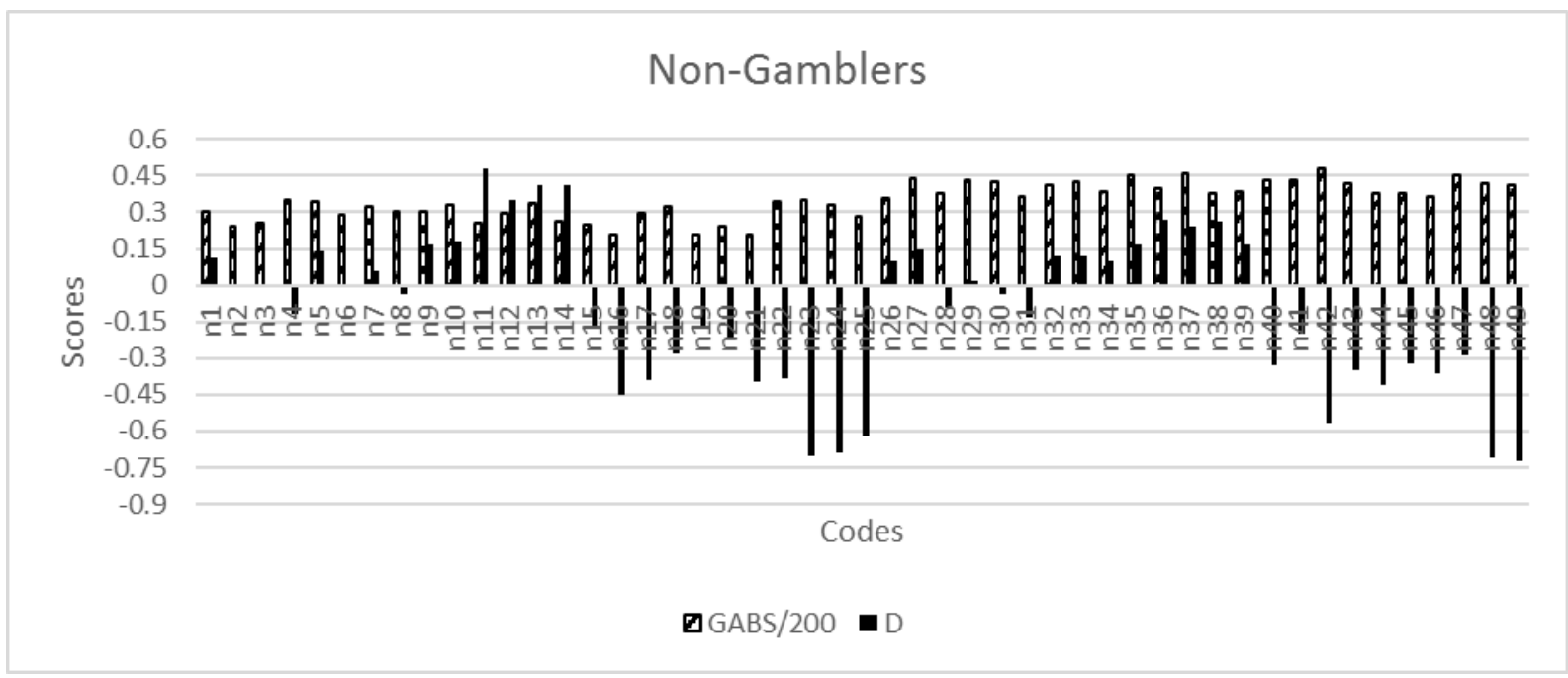

Figure 6 Results of attitudes to gambling measures with explicit method GABS/200 and D. Group "Non-gamblers"

The second research question.

For explicit variable GABS the following percent distribution was obtained.

Social Gamblers: moderate desire to gambling $8 \%$, dependence $84 \%$, heavy dependence on gambling $8 \%$.

Non-Gamblers: moderate desire to gambling $51 \%$, dependence $49 \%$.

Problem Gamblers: moderate desire to gambling $8 \%$, dependence $92 \%$.

One way ANOVA 
Factor «Group» impacts on measured with GABS explicit attitude toward gambling: $\mathrm{F}(2,95)=18.73$, $\mathrm{p}<.001$. The mean of GABS for Social Gamblers exceeds the mean of GABS for Problem Gamblers: LSD $=9.49$, $\mathrm{p}=.014$; the mean for GABS for Social Gamblers exceeds the mean of GABS for NonGamblers: LSD $=19.53, \mathrm{p}<.001$; and the mean of GABS for Problem Gamblers exceeds the mean of GABS for Non-Gamblers: LSD $=10.03$, $\mathrm{p}<.003$ (Fig. 7).

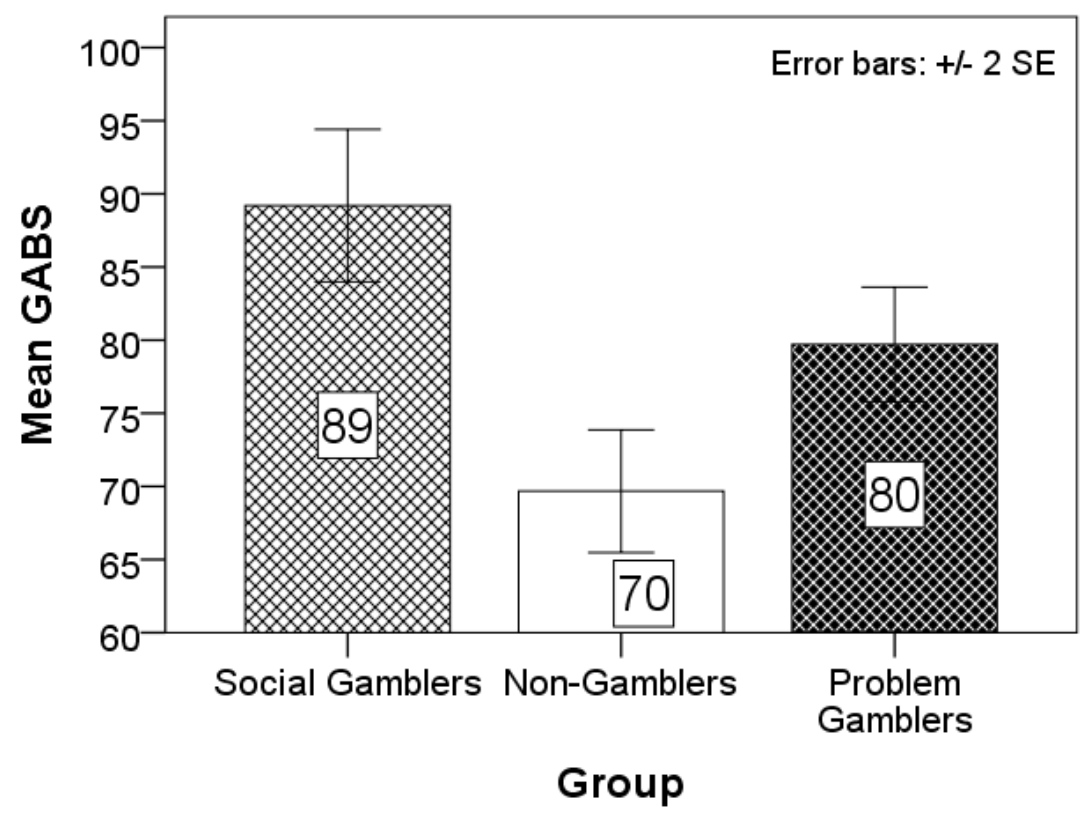

Figure 7 Means of GABS for all groups

The impact of the factor "Group" on the effective size of implicit measured association with gambling was not found: $F(2,95)=0.83$, ns. The comparisons of means and levels of any two groups did not reveal the differences.

\section{The third research question.}

The research of congruence of measurement results, obtained with experimental procedures of the SC-IAT (variable D) and self-reported procedures (variable GABS) was performed using Pearson's correlation coefficients between GABS and positive values of $\mathrm{D}$, as well as by calculating the percent of matching results.

Pearson's correlation coefficients.

The results showed compliance of implicit and explicit measurements of researched constructs evaluated by the correlation coefficients, the values of which fall within the valid range from .12 to .72 (Rudman, 2011). The correlation coefficients from this interval were not found. 
Calculation of percent.

To calculate percent match the frequency analysis was used. The following results will match: (1) D "no effect" and GABS "low" or (2) D "medium \& high positive effect size" and GABS "dependence \& heavy dependence".

Obtained percentage of matches: in Social Gamblers: $24.0 \%, \mathrm{n}=25$; in Non-Gamblers: $22.5 \%, \mathrm{n}=49$; in Problem Gamblers: $12.5 \%, \mathrm{n}=24$.

Correlation between $D$ and GABS.

For positive values of $\mathrm{D}$ a negative correlation between implicit and explicit measures was found: $\mathrm{r}(45)=-.294, \mathrm{p}<.05$. The correlation is mostly pronounced among non-gamblers: $\mathrm{r}(20)=-.505, \mathrm{p}<.05$. Among problem gamblers $\mathrm{r}(13)=-.233, \mathrm{p}=.444$. When $\mathrm{D} \leq-0.15$ among non-gamblers $\mathrm{r}(12)=-.668, \mathrm{p}<.05$.

It is possible that this indicates a pursuit (mainly among non-gamblers and problem gamblers) to hide the internal propensity to gambling during explicit measures.

For negative associations with gambling the statistically significant association was not found.

With negative $\mathrm{D}$ in the group of social gamblers $\mathrm{r}(12)=-.242, \mathrm{p}=.448$, in non-gamblers $\mathrm{r}(29)=-.249, \mathrm{p}=.192$, in problem gamblers $\mathrm{r}(11)=.406$, $\mathrm{p}=.215$. The positive correlation may indicate that with the increase of negative associations with the game among problem gamblers the explicit scores of the GABS decrease. Negative correlations may suggest that with the increase of negative associations with the game among problem gamblers the explicit scores still increase.

\section{Conclusions and Discussion}

As a result of the research, its aim has been implemented and the main results are presented. The main provisions of the methodology of implicit social cognition give reasons to believe that the measurements with the use of implicit and explicit measures of attitudes towards gambling provide more deep understanding of psychological construct (gambling).

Similar results were received in Yi and Kanetkar (2010) research. In the study of Yi \& Kanetkar (2010) a sample of students was selected. It has been found that all of them could have negative attitudes towards gambling, measured implicitly. Yi suggests that as individuals become more vulnerable to gambling severity, not only positive but also negative automatic gambling associations became stronger. In the study by Bravers et. al. (2013, p. 96, p. 94) in problem gamblers, who were cured in clinics, negative implicit attitudes towards gambling were not found. In our study the sample consisted of social (nonproblem) and problematic gamblers and non-gamblers. 
Inour research both positive and negative implicit gambling associations were found in each of the groups. Among social gamblers the positive and negative associations are equally pronounced. Both among non-gamblers and problem gamblers the negative associations are more pronounced than the positive. Problem gamblers have most expressed negative associations.

Explicit attitudes towards gambling are most pronounced among social gamblers and most not pronounced - among non-gamblers. Differences in implicit associations with gambling between the groups were not found.

The correlation coefficients between GABS and positive values of Dfrom interval $(.12 ; .72)$ were not found. The least percentage of matches between the results of implicit and explicit measures is among problem gamblers.

For participants with positive implicit associations with gambling the following regularities were found. For the entire sample the negative correlation between the results of explicit and implicit measures was revealed. The correlation is most pronounced among non-gamblers. Among problem gamblers, with an increase in the effect's size of implicit associations to gambling the negative relationship becomes very close. It is possible that such a relation points out to a craving to hide the intrinsic propensity to gambling during explicit measurements. This result indirectly supports the conclusion that the results of explicit measurements among problem gamblers are less pronounced than that of the social gamblers.

For participants with negative implicit associations with gambling the statistically significant correlation between the results of explicit and implicit measures was not found.

For participants with negative implicit associations with gambling from the group of social gamblers the authors found a rather weak negative correlation between the results of explicit and implicit measures. Negative correlations may indicate that with an increase in negative associations with gambling among the participants the explicit scores still increase.

For participants with negative implicit associations to gambling from the group of problem gamblers the authors found a statistically non-significant, positive moderate relationship between the results of explicit and implicit measures. The positive correlation may indicate that with an increase in negative implicit associations with gambling the explicit scores by GABS among problem gamblers decreases. As a matter of fact, it is a manifestation of reluctance to play, which is found in the verbal self-report of participants who have strong negative implicit associations.

Despite the fact that gamblers have experienced adverse effects associated with gambling, the implicit associations with gambling may be negative, which gives hope for gambling cessation. 
It is possible that the record of relationship between positive and negative implicit associations and severity of explicit attitudes will contribute to the classification of gamblers with low, moderate and high risk.

It is interesting to analyze individual cases of the participants. Often, on the explicit level a participant (problematic gambler) demonstrates a pronounced attitude towards gambling addiction, but on the implicit level - a negative attitude towards gambling. For example, the participant No. 1, who is a problematic gambler, has the pronounced negative attitude. This is a man of 38 years old, single, with secondary special education. The participant plays slot machines several times a week. Approximately for one year he has been attending the group of Gamblers Anonymous, who meet once a week, and is keen to stop gambling. The presence of the pronounced negative attitude may indicate that despite the existing gambling addiction, this participant has an intrinsic readiness to resist, get rid of gambling addiction.

Limitations. Insufficient samples of gamblers. Use of one-dimensional models.

In the long run, it appears interesting to study the influence of contextual factors on implicit and explicit measurements of attitudes towards gambling.

\section{References}

Ackermann, C. L., \& Mathieu, J. P. (2015). Implicit attitudes and their measurement: Theoretical foundations and use in consumer behavior research. Rechercheet Applicationsen Marketing, 30 (2) 55-77.

Ackermann, C. L., \& Teichert, T. (2015). A conceptual paper on implicit gambling orientation. pp. 1-15. Retrieved from http://www.marketing-trends-congress.com/ archives/2016/pages/PDF/ACKERMANN_TEICHERT.pdf

Ashrafiuon, L., \& Rosenberg, H. (2012). Methods of assessing craving to gamble: A narrative review. Psychology of Addictive Behaviors, 26 (3), 536-549.

Ben-Tovim, D., Esterman, A., Tolchard, B., \& Battersby, M. (2001). The Victorian Gambling Screen. Report prepared for the Gambling Research Panel. Melbourne: Victoria.

Breen, R., \& Zuckerman, M. (1999). Chasing in gambling behavior: Personality and cognitive determinants. Personality and Individual Difference, 27, 1097-1111

Brevers, D., Cleeremans, A., Hermant, C., Tibboel, H., Kornreich, C., Verbanck, P., \& Noël, $X$. (2013). Implicit gambling attitudes in problem gamblers: Positive but not negative implicit associations. Journal of Behavior Therapy and Experimental Psychiatry, $44(1$, 94-97.

De Houwer, J. (2006). Using the implicit association test does not rule out an impact of conscious propositional knowledge on evaluative conditioning. Learning and Motivation, 37, 176-187.

Fazio, R. H. (2007). Attitudes as object-evaluation associations of varying strength. Social Cognition, 25 (5), 603-637.

Ferris, J., \& Wynne, H. (2001). The Canadian problem gambling index: Final report. Submitted for the Canadian Centre on Substance Abuse. Retrieved from https://www.problemgambling.ca/EN/Documents/ProblemGamblingSeverityIndex.pdf 
Irina Plotka, Nina Blumenau, Zhanna Vinogradova. Research of Implicit Attitudes Towards Gambling for Gamblers and Non-Gamblers

South Oaks Gambling Screen (SOGS). Retrieved from http://www.south-oaks.org/ files/South_Oaks_Gambling_Screen.pdf.

George, D., \& Mallery, P. (2003). SPSS for Windows step by step: A simple guide and reference. 11.0 update (4th ed.). Boston: Allyn \& Bacon.

Greenwald, A. G., \& Banaji, M. R. (1995). Implicit social cognition: Attitudes, self-esteem, and stereotypes. Psychological Review, 102 (1), 4-27.

Karpinski, A., \& Steinman, R. B. (2006). The single category implicit association test as a measure of implicit social cognition. Journal of Personality and Social Psychology, 91 (1), 16-32.

Lesieur, H. R., \& Blume, S. B. (1987). The South Oaks Gambling Screen (SOGS): A new instrument for the identification of pathological gamblers. American Journal of Psychiatry, 144 (9), 1184-1188.

Nosek, B. A., Greenwald, A. G., \& Banaji, M. R. (2007). The Implicit Association Test at age 7: a methodological and conceptual review. In J. A. Bargh (coord.), Automatic processes in social thinking and behaviour (pp. 265-292). New York, NY: Psychology Press.

Orford, J., Griffiths, M., Wardle, H., Sproston, K., \& Erens, B. (2009). Negative public attitudes towards gambling: Findings from the 2007 British Gambling Prevalence Survey using a new attitude scale. International Gambling Studies, 9, 39-54.

Petty, R. E., Fazio, R. H., \& Briñol, P. (2009). The new implicit measures: An overview. InR. E. Petty, R. H., Fazio, \& P., Briñol (Eds.), Attitudes: Insights from the new implicit measures (pp. 3-18). New York, NY: Psychology Press.

Plotka, I., Igonin, D., Blumenau, N., Simane, L., Lutikova, N., \& Morozova, M. (2013). Investigation of attitudes toward gambling with implicit association test and selfreported measures. International Journal of Development and Educational Psychology, 1 (2), 597-610.

Rickwood, D., Blaszczynski, A., Delfabbro, P., Dowling, N., \& Heading, K. (2010). The Psychology of Gambling. APS Review Paper. Final. Retrieved from file:///C:/Users/Nina/Downloads/APS-Gambling-Paper-2010\%20(1).pdf

Rudman, L. A. (2011). Implicit Measures for Social and Personality Psychology. Rutgers University, USA: SAGE Publications.

Strack, F., \& Deutsch, R. (2004). Reflective and Impulsive Determinants of Social Behavior. Personality and Social Psychology Review, 8 (3), 220-247.

Validation of the Victorian Gambling Screen. Prepared for the Gambling Research Panel by The Centre for Gambling Research Australian National University. GRP Report No. 7. (2004). Australia: Community support fund. Retrieved from https://digitalcollections. anu.edu.au/bitstream/1885/45190/3/WholeVicGamScr_03.pdf

Yi, S., \& Kanetkar, V. (2010). Implicit measures of attitudes toward gambling: An exploratory study. Journal of Gambling Issues, 24, 140-163.

Wiers, R. W., \& Stacy, A. W. (Eds.) (2006). Handbook of implicit cognition and addiction. Thousand Oaks, CA: SAGE Publishers.

Zack, M., \& Poulos, C. X. (2004). Amphetamine primes motivation to gamble and gamblingrelated semantic networks in problem gamblers. Neuropsychopharmacology, 29, 195207.

Zack, M., \& Poulos, C. X. (2007). A D2 antagonist enhances the rewarding and priming effects of a gambling episode in pathological gamblers. Neuropsychopharmacology, 32, $1678-1686$. 
SOCIETY. INTEGRATION. EDUCATION

Proceedings of the International Scientific Conference. Volume I, May $27^{\text {th }}-28^{\text {th }}$, 2016. 498-514

Kozlov, V. V., \& Karpov, A. A. (2011). Psihologija igrovoj zavisimosti [The psychology of gambling addiction]. Moskva: Psihoterapija. 